\title{
Acknowledgement to the Team of Reviewers in 2020
}

\author{
Shuangshuang Xu, Assistant Editor
}

Published online: 6 May 2021

(c) Springer Nature Switzerland AG 2021

The third year of Human Arenas (HA) has been difficult due to Covid-19 pandemic. At the end of 2020, the editorial team of HA would like to express our gratitude to all our reviewers around the world for their devotion of precious time and expertise to HA's development. Our reviewers' open mind, huge passion, and constructive suggestions have helped HA not only in assessing, inspiring, and cultivating innovative ideas but also in trying to open a creative interdisciplinary zone to reflect and understand the strong impacts of pandemic on human's lives. In the year of 2020, HA has published 69 articles in total and has been included in the journal list of Scopus database. Our huge progress would not be achieved without the help and support of our international reviewers. We sincerely acknowledge all the reviewer's generous contribution and look forward to further cooperation in the coming 2021 with same passion and vision in this special challenging time.

2020 reviewers (in alphabetic order)

Muhammed Abdulai

Joshy Abraham

Jennifer Adler

James Akpan

Charles Alberts

Nicolás Alessandroni

Sana Ali

Fernando Andacht

Molly Andrews

Guilherme Siqueira Arinelli

Danna Aristizabal

Sarah Awad

Michael Bamberg

Parul Bansal

Alicia Barreiro

Bruno Jaraba Barrios

Stephanie Barros

Aleksandr Baucal

Zack Beckstead

Robert Beshara

Thomas Boll 
Silje Bonden

Dany Boulanger

Clint Burnham

Tobias Busch

Stephen Butler

Caitlin Cahill

Erinn Cameron

Marc Antoine Campill

Luna Carpinelli

Brena Carvalho

Chrissie Carvalho

Jorge Castro

Graziano Cecchinato

Nandita Chaudhary

Niklas Alexander Chimirri

Julie Bo Christensen

Janet Conti

Jean Nikola Cudina

João Roberto R. T. da Silva

Marcio Santana da Silva

Nadia Dario

Marcio Nunes de Abreu

Ana Luiza de França Sá

Raffele De Luca Picione

Carmen Dege

Martin Dege

Johanna Lisa Degen

Raffaele De Luca Picione

Maria Gabriela Di Gesu

Lars Schermer Didriksen

Denis Ebbesen

Charbel El-Hani

Danilo Silva Guimarães

Moisès Esteban-Guitart

Sebastian Johann Feil

Chiara Fioretti

Pablo Fossa

Mark Freeman

Paul Galea

Vladimer Lado Gamsakhurdia

Adrian-Mario Gellel

Diana Di Gennaro

Afsaneh Ghanizdeh

Kyoko Murakami

Roberto Musa Giuliano

Ramon Cerqueira Gomes

Davood Gozli

Gemma Maria Griffith

Renato Guimaraes 
Jaakko Hilppö

Yasmin Ibrahim

Maria Impedovo

Robert Innis

Bruno Jaraba Barrios

Mogens Jensen

Annika Jonsson

Line Joranger

Gordana Jovanović

Cristana Kaipper

Eleni Kalantidou

Effie Karageorgos

Kadri Kasemets

Hroar Klempe

Alaric Kohler

Koji Komatsu

Karsten Krauskopf

Surbhi Kumar

Cynthia Langtiw

Maria Beatrice Ligorio

José Luis Linaza

Camila Lisboa

Valentina Luccarelli

Luka Lucic

Maria Lyra

Thomas Madsen

Nupur Mahajan

Jens Mammen

Amelia Manuti

Ana Marjanovic-Shane

Athanasios Marvakis

Noomi Christine Linde Matthiesen

Lucas Mazur

Maria Medved

Enrica Mele

Daiane Menezes

Guenter Mey

Dominik Stefan Mihalits

Juan David Millán

Wenty Marina Minza

Monica Mollo

Karina Moutinho

Kyoko Murakami

Mariann Märtsin

Martin Mølholm

Cathy Nicholson

Kleber Ferreira Nigro

Himmbler Olivares

Jeppe Olsen 
Yasuhiro Omi

Guro Brokke Omland

Julio César Ossa

Kathrin Otrel-Cass

Seth Oppong

Federica Pascarella

Francesco Piro

Radha Pavanasam

María Elisa Molina Pavez

Susan Petrilli

Punya Pillai

Francesco Piro

Anna-Mai Pirttilä-Backman

Seamus Power

Falu Rami

Alan Rayner

Paul Rhodes

Leonardo Rocha

Paul Sebastian Ruppel

Jesse Ruse

Claudio Russo

Khatereh Saghafi

Julio Eduardo Mazorco Salas

Gordon Sammut

Nadia Sansone

Sam Sarpong

Raquel Scartezini

Sanna Schliewe

Ernst Schraube

Hildegunn Marie Tønnessen Seip

Mojdeh Shahnama

Sara Silva

Alfred Bordado Sköld

Corinne Squire

Sujata Sriram

Hendericus Stam

Irene Strasser

Henrik Sætra

Syeda Arifa Tasneem

Floyd Thompkins

Kumru Toktamis

Rosa Traversa

Estefany Trujillo

Tatiana Valerio

Jaan Valsiner

Leonardus A. van Gulik

Anja Visser-Nieraeth

Brady Wagoner

Ryan Wasser 


\section{Crista Weise}

Martin Wieser

Manfred Wu

Publisher's Note Springer Nature remains neutral with regard to jurisdictional claims in published maps and institutional affiliations. 